\title{
Tai Chi for the Prevention of Falls Among Older Adults: A Critical Analysis of the Evidence
}

\begin{abstract}
Samuel R. Nyman
Despite interest as to the benefits of Tai Chi, there remains a controversy over its effectiveness as an exercise intervention for preventing falls among older adults. This review synthesizes the evidence base with a focus on meta-analyses and randomized controlled trials with community-dwelling older adults. It provides a critical lens on the evidence and quality of the trials. Highquality evidence suggests that Tai Chi is an effective intervention for preventing falls in community settings; however, there is unclear evidence for long-term care facilities and an absence of evidence for hospital settings. When compared directly with other exercise interventions, Tai Chi may offer a superior strategy for reducing falls through its benefits on cognitive functioning. Using data from the current Cochrane review, a new synthesis is presented suggesting that $71-81 \%$ of community-dwelling older adults are adherent to class-based Tai Chi interventions. The practical opportunities and challenges for practitioners are discussed.
\end{abstract}

Keywords: accidental fall, exercise, meta-analysis, randomized controlled trial, review

Falls among older adults have been researched since the 1940 s and are internationally recognized as a public health issue (Speechley, 2011; World Health Organization, 2007). For community-dwelling adults aged 65 and above, approximately one in three fall each year, and this frequency increases to one in two among those aged 80 and over and among residents of long-term care institutions (Martin, 2011; Rubenstein, 2006). Falls are the leading cause for emergency department presentation in adults aged 65 and over (Samaras, Chevalley, Samaras, \& Gold, 2010) and are a significant risk for morbidity, mortality, and increased health care costs (Burns, Stevens, \& Lee, 2016). Older adults can also develop a fear of falling that leads to activity limitation, social isolation, and a reduction in quality of life (Peel, 2011; World Health Organization, 2007). Several interventions have been developed to reduce the risk of falls among older adults (Gillespie et al., 2012). These include single interventions (e.g., exercise), multiple interventions (combinations of interventions such as exercise and home safety modifications), and multifactorial interventions (customized interventions depending on the needs of the individual using a menu of intervention options). For single interventions, exercise has been identified as the most effective approach for preventing falls (Gillespie et al., 2012). It has also been suggested that single interventions are as effective as multifactorial interventions for populations at risk of falls and may well be more acceptable and cost effective (Campbell \& Robertson, 2007).

In this context, Tai Chi has been researched as a strategy with great potential for preventing falls among older adults (Nyman \&

(C) 2021 The Author. Published by Human Kinetics, Inc. This is an Open Access article distributed under the terms of the Creative Commons Attribution 4.0 International License, CC BY 4.0, which permits unrestricted noncommercial and commercial use, distribution, and reproduction in any medium, provided the original work is properly cited, the new use includes a link to the license, and any changes are indicated. See https://creativecommons.org/licenses/by/4.0/. This license does not cover any third-party material that may appear with permission in the article.

Nyman (snyman@bournemouth.ac.uk) is with the Department of Medical Science and Public Health, Faculty of Health and Social Sciences, Bournemouth University, Poole, United Kingdom.
Skelton, 2017). Tai Chi has been practiced widely in China and Asian cultures and has become more popular in western countries. Tai Chi has been recommended for older adults to meet U.K. guidelines for participation in physical activity (Department of Health and Social Care, 2019) and is recommended by Public Health England (Foster et al., 2018) and the National Health Service (NHS), particularly for inactive older adults (NHS Choices, 2018). However, there remains a controversy with regard to the health benefits of Tai Chi and, in particular, its effectiveness in preventing falls among older adults.

This narrative review aims to critically synthesize the evidence for Tai Chi for the prevention of falls among older adults. This review will focus on gold-standard evidence-meta-analyses of randomized controlled trials-most of which has been conducted with community-dwelling older adults. For the first time, I will bring together in one place a critical analysis of the fragments of information from different reviews in order to provide a comprehensive review of the evidence and the quality of the evidence base and a new synthesis on adherence levels by older adults to Tai Chi classbased exercise interventions. I will also include studies that have compared the effectiveness of Tai Chi for the prevention of falls with other exercise-based interventions. I will then conclude with the practical implications for exercise practitioners. While there are existing meta-analyses on this subject, they have not been synthesized for an overall interpretation of the evidence base, particularly in relation to adherence, different populations, and the effectiveness of Tai Chi relative to other exercise interventions. Before reviewing the evidence base, I will first describe Tai Chi exercise.

\section{Tai Chi}

Tai Chi (also written taiji, t'ai ji, or similar) is an ancient form of Chinese mind-body exercise in which participants carry out slow, smooth, and continuous body movements along with deep breathing and mental concentration (Lee, Lee, \& Woo, 2010); it is equivalent to moderate-intensity exercise and quiet meditation (Li, Hong, \& Chan, 2001). Thus, as a mind-body exercise, it incorporates both a form of moving meditation with gentle strengthening of muscles and balance control. In describing Tai Chi we must 
discuss its departure from Tai Chi chuan and it being a form of qi gong. Tai Chi chuan (also written taijiquan, t'ai ji quan, or similar) is a martial art. Tai Chi can be translated as "grand ultimate" and chuan translated as "fist" (or similar wording) (Wayne \& Fuerst, 2013). Tai Chi chuan is a martial art that incorporates the Chinese philosophy of Tai Chi (Chang, Nien, Tsai, \& Etnier, 2010) but is otherwise a form of boxing or similar exercise. The Tai Chi that is discussed in this review is not a martial art but a gentle exercise designed to holistically promote health for both mind and body.

The Chinese philosophy that underpins Tai Chi has much in common with qi gong; hence, many consider Tai Chi to be a form of qi gong (Wayne \& Fuerst, 2013). Qi gong is an umbrella term for ancient Chinese traditional practices of self-cultivation and energy preservation (Acton, 2009). Qi can be translated as "vital energy," "breath," or "spirit" (Wayne \& Fuerst, 2013). Qi gong is thus energy work, whereby practitioners seek to cultivate the optimal conditions for their qi in order to provide nourishment and preservation. Under this framework, qi is considered a unified energy; everyone holds energy/qi and is connected to each other and to the energy in the universe (Acton, 2009). Illness can be considered an imbalance of energy somewhere in the body. Qi qong then targets the body, breath, and mind in order to bring each into harmony and, therefore, bring qi into harmony to sustain health, joy, and longevity (Acton, 2009). Many are familiar with the related Chinese philosophy of yin and yang. These represent two ultimate, opposing, and interdependent forces (e.g., dark and light, female and male, low and high, slow and fast, inhale and exhale). The interactions between yin and yang are proposed to make the universe and humans function energetically. It is this philosophy that underpins qi gong and Tai Chi movements (Acton, 2009). Therefore, Tai Chi aims to relax, strengthen, and integrate the mind and body to enhance the flow of qi (Wayne \& Fuerst, 2013). In so doing, one can achieve "eternal spring," and along with longevity, retain health, vigor, and mental capacity well into one's later years (Acton, 2009).

A complication in defining Tai Chi is that it has many elements. This makes it difficult to isolate a particular mechanism for Tai Chi to provide health benefits. Eight elements have been identified as follows: focused attention, imagery and visualization, enhanced integration of physiological systems, moving meditation, strength and flexibility training, more efficient breathing, social support from attending classes, and a vehicle for increased spirituality (Wayne \& Fuerst, 2013).

\section{The Evidence for Tai Chi for Preventing Falls}

\section{Current Evidence From an Overview of Systematic Reviews and Two Meta-Analyses}

Recently, an overview of 14 systematic reviews was published on the effectiveness of Tai Chi for improving postural balance and preventing falls (Zhong et al., 2020). Ten systematic reviews were published in English and four in Chinese. Eight focused on older adults, and the remainder focused on specific patient groups: Parkinson's disease, stroke, heart failure, or osteoarthritis patients. The authors were largely critical of the quality of the existing body of systematic reviews. Although they suggested that the systematic reviews were largely complete in their reporting, they found only nine of 14 systematic reviews to be of low risk of bias, and one was of low and 13 of critically low methodological quality. However, only four of the systematic reviews provided meta-analyses of the effectiveness of Tai Chi on reducing the rate of falls among older adults. Of these, one was old (Logghe et al., 2010) and another could not be located despite full citation details being given (likely published in Chinese; Zheng, Zhang, \& Tong, 2013). The remaining two meta-analyses were both published in 2017 and deserve attention (Huang, Feng, Li, \& Lv, 2017; Lomas-Vega, ObreroGaitán, Molina-Ortega, \& Del-Pino-Casado, 2017) (see Table 1).

The meta-analysis by Huang et al. (2017) was on the benefits of Tai Chi for older adults, including the general healthy population and those at risk of falls. Their meta-analysis suggested that Tai Chi reduces the rate of falls on average by $31 \%$ (incident rate ratio $[\mathrm{IRR}]=0.69,95 \%$ confidence interval $[\mathrm{CI}],[0.60,0.80], 15$ trials) and the number of people falling at least once by $20 \%$ (IRR $=0.80$, $95 \%$ CI [0.72, 0.88], 16 trials). Huang et al. (2017) found the risk of bias of the included trials to be unclear or low, and sensitivity analyses by sample size, risk of bias, or comorbidity showed no major influence on the primary results. However, they did note that there may be publication bias, because the funnel plots showed asymmetry (studies with null effects were less likely to be published). Despite this glowing report on the effects of Tai Chi in terms of magnitude, consistency, and quality of the evidence, the overview authors were much more conservative. They found the Huang et al. review to be of "critically low" methodological quality (Zhong et al., 2020). However, it ticked 12 out of $16(75 \%)$ boxes on a measurement tool to assess systematic reviews (AMSTAR) 2 checklist. The deductions were for not reporting a preestablished protocol, the study designs for inclusion, list of excluded studies with justifications, and for not reporting on the source of funding for the included trials (although this would be unlikely to be a significant source of bias). While the risk of bias for the review was marked with a smiley face and 25 out of 27 boxes were ticked for reporting (preferred reporting items for systematic reviews and meta-analyses [PRISMA] checklist), it was marked as only providing moderate-grade evidence due to the suspicion of publication bias. This seems to be an overly negative assessment of the quality of evidence from this review.

The meta-analysis by Lomas-Vega et al. (2017) was also on the benefits of Tai Chi for older adults, including the general healthy population and those at risk of falls. Their meta-analysis suggested that Tai Chi reduces the rate of falls on average by $43 \%$ in the first 11 months (IRR $=0.57,95 \%$ CI [0.46, 0.70], five trials) and $13 \%$ from 12 months onward (IRR $=0.87,95 \%$ CI [0.77, 0.99], six trials). The mean of these two pooled estimates (of $43 \%$ and $13 \%=28 \%$ ) was approximately that estimated by Huang et al. (2017) at $31 \%$. However, the lower pooled falls rate at 12 months or more of follow-up was perhaps underestimated, because the rates provided in two trials were higher than those reported by LomasVega et al. More specifically, the significant incidence rate ratio of 0.32 (95\% CI [0.14, 0.71]) at 18 months (Hwang et al., 2016) was higher than the null reported by Lomas-Vega et al. $(0.80,95 \% \mathrm{CI}$ $[0.64,1.01])$, and the significant rate ratio (RR) of $0.74(95 \% \mathrm{CI}$ $[0.56,0.98])$ at 12 months (Tousignant et al., 2013), was higher than the null reported by Lomas-Vega et al. $(\mathrm{RR}=0.87,95 \% \mathrm{CI}$ $[0.69,1.10])$. The results of another trial were difficult to explain; the falls rate over 17 months from a 20 -week intervention period showed a reduction in falls of $58 \%$ across all trial arms with no difference between the control group and the two Tai Chi groups (classes either once or twice weekly; Taylor et al., 2012). However, $60 \%$ of the sample continued with exercise after the interventions ceased, and so the fall reductions may have been the result of exercise conducted outside the interventions. One study was conducted with older adults from care homes (Faber, Bosscher, Chin, \& van Wieringen, 2006; this population will be discussed subsequently), and the study with the poorest results for Tai Chi was 
Table 1 Summary of the Systematic Reviews Synthesized That Assessed the Effectiveness of Tai Chi to Prevent Falls

\begin{tabular}{llll}
\hline Author (date) & Focus of the review & Main results & Strengths and weaknesses of the review \\
\hline $\begin{array}{l}\text { Overview of 14 systematic reviews } \\
\text { Zhong et al. (2020) }\end{array}$ & $\begin{array}{l}\text { Postural balance and fall } \\
\text { prevention among the } \\
\text { healthy older adult } \\
\text { population and specific } \\
\text { patient groups }\end{array}$ & $\begin{array}{l}\text { Existing systematic reviews have a high } \\
\text { risk of bias. It is impossible to draw } \\
\text { conclusions as to the effectiveness of Tai }\end{array}$ & $\begin{array}{l}\text { Provides an overview of 14 reviews and assesses their } \\
\text { quality in several dimensions. However, on inspection } \\
\text { of the two most recent meta-analyses by Huang et al. } \\
\text { (2017) and Lomas-Vega et al. (2017), the quality } \\
\text { assessment is overly negative. }\end{array}$
\end{tabular}

Meta-analyses

Huang et al. (2017) Rate of falls and number of people falling at least once among the healthy older adult population and those at risk of falls

Tai Chi reduces the rate of falls $(\mathrm{IRR}=0.69,95 \%$ CI $[0.60,0.80], 15$ trials) and the number of people falling at least once (IRR $=0.80,95 \% \mathrm{CI}$ [0.72, 0.88], 16 trials).

Lomas-Vega et al. Rate of falls among the (2017) healthy older adult population and those at risk of falls

Tai Chi reduces the rate of falls in the first 11 months $(\mathrm{IRR}=0.57,95 \% \mathrm{CI}$ $[0.46,0.70]$, five trials) and from 12 months onward (IRR $=0.87,95 \% \mathrm{CI}$ [0.77, 0.99], six trials).

Sherrington et al. Rate of falls and number (2019) of people falling at least once among communitydwelling older adults

Sherrington et al. Further analysis to (2017) Sherrington et al. (2019)

$\mathrm{Ng}$ et al. (2019)

Further analysis to Sherrington et al. (2019)

Cameron et al. (2018)

Rate of falls and number of people falling at least once among older adults in care facilities and hospital settings

Del-PinoCasado, ObreroGaitán, and Lomas-Vega (2016)

Rate of falls among older adults who are frail or at risk of falls
Tai Chi reduces the rate of falls $(\mathrm{RR}=0.81,95 \%$ CI [0.67, 0.99], seven trials, low-certainty evidence) and the number of people falling at least once $(R R=0.80,95 \%$ CI [0.70, 0.91], eight trials, high-certainty evidence).

Exercise interventions reduce the rate of falls, and reduce them to a greater extent if they challenge balance and are of a dose of $3 \mathrm{hr}$ or more per week $(\mathrm{IRR}=0.61,95 \%$ CI $[0.53,0.72], 88$ trials). Tai Chi is one way of providing a safe means of challenging balance.

Tai Chi is one of three types of intervention programs that are effective in reducing falls (on average delivered over 20 weeks, $71 \%$ group based, $7 \%$ tailored)

No evidence for Tai Chi for preventing falls in long-term care facilities (three trials) and an absence of evidence in hospital settings.

Tai Chi reduces the rate of falls (absolute risk reduction $=-0.10,95 \%$ CI $[-0.06$, $-0.15], 13$ trials)
Robust review, which acknowledged there may be publication bias. Zhong et al. (2020) identified these methodological limitations of the review: Not reporting a preestablished protocol, the study designs for inclusion, list of excluded studies with justifications, and for not reporting on the source of funding for the included trials.

Robust review that separated the effectiveness of Tai Chi by length of follow-up. Zhong et al. (2020) identified these methodological limitations of the review: Not reporting a preestablished protocol, the study designs for inclusion, list of excluded studies with justifications, for not reporting on the source of funding for the included trials, and for a partially comprehensive literature search strategy.

Gold standard, robust, systematic review, with overall pooled effect. However, the pooled effect does not take into consideration important factors such as adherence, length/dose of intervention, and duration of follow-up.

Gold standard, robust, systematic review, with overall pooled effect that accounts for important moderator variables. However, the effect of Tai Chi could not be isolated from their report.

Gold standard, robust, systematic review, with overall pooled effect. However, finer-grained analysis of the influence of program factors within each type of intervention on falls (such as dose of Tai Chi) was not conducted.

Gold standard, robust, systematic review. However, the pooled effect was for exercise interventions overall and was not calculated for Tai Chi in isolation. Also, the pooled effect did not take into consideration important factors such as adherence, length/dose of intervention, and duration of follow-up.

A well-conducted review on important patient groups. However, absolute risk reduction is a measure rarely used in the fall prevention literature and so not readily comparable (rather rate of falls and number of people falling at least once). The authors stated that their results equated to a reduction in the risk of falling by $21 \%$ (95\% CI $[12 \%, 30 \%])$. The authors conceded they could not calculate the effect of Tai Chi on rate of falls due to insufficient data. The authors concluded that a minimum dose of $60 \mathrm{~min}$ of Tai Chi weekly for 12 weeks or more appears effective, although this will need to be confirmed. 
Table 1 (continued)

\begin{tabular}{|c|c|c|c|}
\hline Author (date) & Focus of the review & Main results & Strengths and weaknesses of the review \\
\hline $\begin{array}{l}\text { Winser, Tsang, } \\
\text { Krishnamurthy, } \\
\text { and Kannan (2018) }\end{array}$ & $\begin{array}{l}\text { Rate of falls among } \\
\text { people with Parkinson's } \\
\text { disease and who have } \\
\text { experienced a stroke }\end{array}$ & $\begin{array}{l}\text { Tai Chi reduces the rate of falls among } \\
\text { people with Parkinson's disease } \\
(\mathrm{OR}=0.47,95 \% \text { CI }[0.29,0.77], \text { three } \\
\text { trials) and who have experienced a } \\
\text { stroke (OR }=0.21,95 \% \text { CI }[0.09,0.48] \text {, } \\
\text { one trial) }\end{array}$ & $\begin{array}{l}\text { A review on important patient groups. However, the } \\
\text { findings are based on a very small number of trials and } \\
\text { ORs are rarely used in the fall prevention literature and } \\
\text { so not readily comparable (rather rate of falls and } \\
\text { number of people falling at least once). Zhong et al. } \\
\text { (2020) identified these methodological limitations of } \\
\text { the review: Not reporting the study designs for } \\
\text { inclusion, using a partially comprehensive literature } \\
\text { search strategy, not reporting a list of excluded studies } \\
\text { with justifications, the source of funding for the } \\
\text { included trials, not assessing risk of bias in included } \\
\text { studies, and not assessing risk of publication bias. }\end{array}$ \\
\hline
\end{tabular}

Note. Reviews are listed in the order of presentation in the main text. $(\mathrm{I}) \mathrm{RR}=$ (incident) rate ratio; $\mathrm{OR}=$ odds ratio; $\mathrm{CI}=$ confidence interval.

from a low-dose (13-week) intervention with low adherence (47\% attended $80 \%$ or more of the sessions) (Logghe et al., 2009).

Lomas-Vega et al. (2017) reported a similar assessment of highquality evidence from the included trials. However, they did note that there was a high risk of attrition bias in two trials and in the long-term follow-up of another. With this glowing report on the short-term effects of Tai Chi in terms of magnitude, consistency, and quality of the evidence, the overview authors were not as unfavorable with their assessment of the quality of evidence as with the Huang et al. review. They found the Lomas-Vega et al. review to be of "critically low" methodological quality. However, it ticked 11.5 out of $16(72 \%)$ boxes on the AMSTAR 2 checklist. The deductions were for the same items as for Huang et al.'s review, except that the Lomas-Vega et al. review had a partial tick for a comprehensive literature search strategy. The risk of bias for the review was marked with an unhappy face. However, it ticked 24.5 out of 27 for reporting (PRISMA checklist) and was marked as providing high-grade evidence.

The overview authors' conclusions were in the main two points: future systematic reviews need to be better conducted in order to reduce the risk of bias (not risk of bias within the trials reviewed), and they found it impossible to draw conclusions as to the effectiveness of Tai Chi for preventing falls. However this was an overly cautious conclusion from an overly negative assessment of review quality, because the Huang et al. (2017) and Lomas-Vega et al. (2017) meta-analyses provide clear results with regard to the effectiveness of Tai Chi for preventing falls from high-quality evidence.

\section{Evidence From the Current Cochrane Review: Community-Dwelling Older Adults}

The current Cochrane review of exercise-based interventions for reducing falls among community-dwelling older adults was published by Sherrington et al. (2019). Overall, the authors found exercise to reduce the rate of falls by an average of $23 \%(\mathrm{RR}=0.77$, 95\% CI [0.71, 0.83], 59 trials, high-certainty evidence) and the number of people falling at least once by an average of $15 \%$ $(\mathrm{RR}=0.85,95 \%$ CI $[0.81,0.89], 63$ trials, high-certainty evidence). There was no evidence of a difference in effect on both falls outcomes according to whether trials selected participants at increased risk of falling or not. In the subgroup analyses by type of exercise, they found that Tai Chi reduced falls by an average of $19 \%(\mathrm{RR}=0.81,95 \% \mathrm{CI}[0.67,0.99]$, seven trials, low-certainty evidence) and the number of people falling at least once by $20 \%$ $(\mathrm{RR}=0.80,95 \% \mathrm{CI}[0.70,0.91]$, eight trials, high-certainty evidence). This was lower by $5 \%$ for rate of falls but higher by $7 \%$ for number of fallers than the effect seen in the most frequent type of exercise: balance and functional exercise with $24 \%$ reduction in falls $(\mathrm{RR}=0.76,95 \% \mathrm{CI}[0.70,0.81], 39$ trials, high-certainty evidence $)$ and $13 \%$ reduction in number of people falling at least once $(\mathrm{RR}=0.87,95 \% \mathrm{CI}[0.82,0.91], 37$ trials, high-certainty evidence $)$. However, one notices that many more studies have been conducted using balance and functional exercise. In addition, the certainty of evidence for rate of falls for Tai Chi trials was downgraded by two levels due to inconsistency (there was substantial heterogeneity; $I^{2}=74 \%$ ) and risk of bias (removing studies with high risk of bias in one or more items had a marked impact on results).

The same authors conducted supplementary reviews to identify the characteristics of interventions that have larger effects on preventing falls. They found that exercise as a single intervention among community-dwelling older adults reduced falls by an average of $21 \%$ ( 88 trials) and by $39 \%$ ( 88 trials) if the intervention challenged balance and was of a dose of $3 \mathrm{hr}$ or more of weekly exercise (Sherrington et al., 2017). They highlight that Tai Chi is one way of providing a safe means of challenging balance. Indeed, in another review paper of 108 trials, Tai Chi was highlighted as one of three types of intervention programs that are effective in reducing falls (on average delivered over 20 weeks, $71 \%$ group based, $7 \%$ tailored; $\mathrm{Ng}$ et al., 2019). Taken together, the 2019 Cochrane review and supplementary reviews of exercise as a single intervention among community-dwelling older adults found that Tai Chi is among the most effective strategies for preventing falls. This was despite relatively fewer trials having been published on the effectiveness of Tai Chi for preventing falls and lower-certainty evidence in terms of the rate of falls.

In comparing the current Cochrane and supplementary reviews with the aforementioned Huang et al. (2017) and Lomas-Vega et al. (2017) meta-analyses, there are similarities and dissimilarities (see Table 1). The Cochrane and supplementary reviews enable comparison of the effectiveness of Tai Chi relative to other exercisebased interventions, whereas the Huang and Lomas-Vega reviews provide more detailed analyses of Tai Chi in isolation. The Huang and Lomas-Vega reviews presented complementary results (the latter only reported rate of falls, but separated by length of followup), and so only the Huang et al. results are compared with Sherrington et al. (2019) for Tai Chi to reduce the number of people falling at least once and rate of falls. For the number of people falling at least once, both reviews found Tai Chi to be effective, with a pooled estimate of a $20 \%$ reduction (both IRR $=$ $0.80 ; 95 \%$ CI $[0.72,0.88]$ Huang et al. and 95\% CI [0.70, 0.91] Sherrington et al.). This result was despite the Huang et al. metaanalysis including double the number of trials in Sherrington et al. (16 to 8). For the rate of falls, while both reviews found Tai Chi to be effective, they differed in the estimated pooled effect size (31\% 
vs. $19 \%$ : Huang et al. IRR $=0.69 ; 95 \%$ CI $[0.60,0.80]$; Sherrington et al. IRR $=0.81,95 \%$ CI $[0.67,0.99])$. The review by Huang et al. included double the number of trials as Sherrington (15 to 7). It appears that Huang et al. included more trials than Sherrington et al. because they included studies that recruited participants at risk of falls (due to Parkinson's disease, frailty, or stroke). This means that the review by Sherrington et al. provides a clearer picture of the effect of Tai Chi for preventing falls among the general community-dwelling older adult population. Given that Huang et al.'s pooled estimate was higher, this suggests that Tai Chi may be more effective in reducing the rate of falls among older adults in the community at an increased risk of falling. While Huang et al. conducted a subgroup analysis that suggested that Tai Chi is more effective in reducing falls among those not at risk of falling at trial enrollment, it is unclear how they identified fall risk. Indeed, it is noted that patients who are at a higher risk of falls (due to Parkinson's disease, stroke, or institutional dwelling) were included in the group deemed not at risk. Therefore, whether Tai Chi is more or less effective in reducing the rate of falls among at-risk populations is a question for future investigation.

\section{Evidence for Other Populations}

While there is strong, high-quality evidence for exercise-based interventions, including Tai Chi, for preventing falls among community-dwelling older adults, the evidence for older adults residing in institutional settings is rather different. The current Cochrane review of interventions for reducing falls among older adults in care facilities and hospital settings was published by Cameron et al. (2018) (see Table 1). Overall, the authors found null results from very low-quality evidence in care facilities for exercise to reduce the rate of falls ( $\mathrm{RR}=0.93,95 \% \mathrm{CI}[0.72,1.20], 10$ trials, very low-quality evidence) or fallers ( $\mathrm{RR}=1.02,95 \% \mathrm{CI}[0.88,1.18], 10$ trials, lowquality evidence). Similarly, the authors found very low-quality evidence for additional physiotherapy on rehabilitation wards to have a null effect on the rate of falls $(\mathrm{RR}=0.59,95 \% \mathrm{CI}[0.26,1.34]$, two trials) but a potential effect on fallers $(\mathrm{RR}=0.36,95 \% \mathrm{CI}[0.14$, 0.93], two trials). While no Tai Chi trials were conducted in hospitals, three trials were conducted in care facilities. Two trials reported null findings, although they were limited by low adherence (at 24\%; Nowalk, Prendergast, Bayles, D’Amico, \& Colvin, 2001) or shortterm follow-up with a small sample (12 weeks with 59 participants; Choi, Moon, \& Song, 2005). The remaining study was a pilot with a small sample that showed promise for the benefits of Tai Chi (Saravanakumar, Higgins, van der Riet, Marquez, \& Sibbritt, 2014).

Therefore, at present, there is no evidence for Tai Chi for preventing falls in long-term care facilities, and there is an absence of evidence for hospital settings. Perhaps the null findings to date with older adults residing in institutional settings reflect the high level of frailty in these populations. It has previously been reported that Tai Chi may be of less benefit to frailer older adults (Liu \& Frank, 2010). As with exercise in general, if Tai Chi has to be modified to allow those with poor balance to participate by making it seated or removing the need to transfer weight between legs, then it may no longer be beneficial in preventing falls (Skelton \& Mavroeidi, 2018). Nonetheless, a systematic review of trials with older adults who are frail or at risk of falls found Tai Chi to be effective for preventing falls (absolute risk reduction $=-0.10,95 \%$ CI $[-0.06,-0.15], 13$ trials; Del-Pino-Casado et al., 2016). Another systematic review of Tai Chi found that it prevents falls among people with Parkinson's disease (odds ratio $[\mathrm{OR}]=0.47,95 \% \mathrm{CI}[0.29,0.77]$, three trials) and among those who have experienced a stroke $(\mathrm{OR}=0.21,95 \% \mathrm{CI}[0.09$, 0.48], one trial) (Winser et al., 2018) (see Table 1).

\section{The Evidence for Tai Chi for Preventing Falls Relative to Other Exercise Interventions}

It is believed that the mechanism by which exercise interventions prevent falls is by increasing standing and dynamic balance, strength and power, and coordination (Skelton \& Mavroeidi, 2018). While previous reviews have highlighted that there is more than one way to achieve these objectives ( $\mathrm{Ng}$ et al., 2019; Sherrington et al., 2017), few studies have directly compared whether some exercise interventions are more effective than others in preventing falls. The Cochrane review of exercise-based interventions for preventing falls among community-dwelling older adults found little evidence for head-to-head comparisons of types of interventions. The authors reported no meta-analyses and low-certainty evidence (Sherrington et al., 2019).

The earliest trial to compare Tai Chi with another exercise intervention was published in the 1990s. Compared with computerized balance training, Tai Chi was found to have reduced time to first fall, which could be due to several benefits measured (grip strength, systolic blood pressure, fear of falling, perceived ability to do all that they would like to do, and distance walked in 12 min; Wolf et al., 1996). A trial reported no benefit of Tai Chi on reducing falls relative to resistance exercise or usual care, but this study was underpowered for detecting a lower rate of falls relative to the resistance exercise $(-15 \%)$ or usual care groups $(-27 \%)$ (Woo, Hong, Lau, \& Lynn, 2007). Other trials have reported similar benefits on several measures of physiological falls risk between Tai Chi and otago exercise (Son, Ryu, Jeong, Jang, \& Kim, 2016) and between Tai Chi, yoga, and balance training (Ni et al., 2014). Another trial reported a greater reduction in falls and use of drug therapy among those with early or mild Parkinson's disease relative to generic exercise (aerobic, dance, and treadmill training; Li, Liu, Dai, \& Dai, 2020). Furthermore, a trial in Canada found Tai Chi to have a $26 \%$ greater reduction in falls than physiotherapy with frail older adults from the community (Tousignant et al., 2012). The only difference they found to explain the greater reduction of falls from Tai Chi was that the physiotherapy group had a reduction in general self-efficacy.

What is illuminating in the few studies that have performed a head-to-head comparison of Tai Chi with another exercise intervention is two studies that included a measure of cognition. A recent trial with older adults at high risk of falling compared Tai Chi with multimodal exercise and a stretching control arm. While both exercise interventions reduced falls relative to the control arm, the Tai Chi intervention had a greater effect size and a $31 \%($ IRR $=0.69,95 \% \mathrm{CI}$ $[0.52,0.94])$ greater reduction in falls compared with the multimodal exercise arm at 6 months ( $\mathrm{Li}$ et al., 2018). Further, at the 12-month follow-up, the Tai Chi group had a 53\% (IRR $=0.47,95 \%$ CI [0.24, 0.92]) greater reduction in serious injurious falls compared with the multimodal exercise group (Li, Harmer, Eckstrom, et al., 2019). Their analysis of secondary outcomes suggested that the superiority of Tai Chi was due to its greater ability, relative to multimodal exercise, to increase dual-task walking (Li, Harmer, \& Chou, 2019).

A trial in Taiwan compared home-based Tai Chi with lowerextremity training among older adults that attended an accident emergency department with a fall-related injury within the past 6 months (Hwang et al., 2016). The Tai Chi group had a 68\% $(\mathrm{IRR}=0.32,95 \% \mathrm{CI}[0.14,0.71])$ greater reduction in falls compared with the lower-extremity training group over the 18-month study. The authors' analysis found that the Tai Chi group improved cognition and, in particular, suggested that its effect on executive functioning was key (Hwang et al., 2016). 
Therefore, there is little evidence to draw from as to whether Tai Chi is superior to other exercise interventions for preventing falls, and further research is required into the mechanisms by which Tai Chi improves health (Hackney \& Wolf, 2014). Nonetheless, there is emerging evidence that Tai Chi may be more effective because it is better able to improve cognition among older adults. This could mean that Tai Chi would be particularly beneficial among older adults with a cognitive impairment such as dementia. Only one randomized controlled trial to date has been published testing the benefits of Tai Chi with community-dwelling older adults with dementia (Nyman et al., 2019). Although the study was not powered to test for a reduction in falls, it found strong promise for Tai Chi to reduce falls and maintain quality of life among people with dementia. Surprisingly, no benefits were identified on balance or cognition outcomes, although this may have been due to the low average adherence to home-based practice during the 20 -week intervention $(47 \%)$. The study also found that falls need to be recorded more intensively with older adults with dementia than with their peers without cognitive impairment (Adamczewska et al., 2019).

\section{The Quality of the Evidence Base on the Health Benefits of Tai Chi in General}

Given the multiple factors that may increase the risk of a fall by an older adult (Martin, 2011), of relevance is the potential for Tai Chi to alleviate the symptoms of other health conditions that may increase the risk of falling. Unfortunately, the quality of trials conducted on the benefits of Tai Chi for health more broadly (in other areas besides falls) has been widely criticized. While one umbrella review (overview of systematic reviews) did not include the quality of trials in their assessment of the evidence (Huston \& McFarlane, 2016), another umbrella review that did relayed the concerns of the review authors with regard to the methodological limitations of trials and the insufficient number of studies (Solloway et al., 2016). Indeed, systematic review authors have reported low-quality evidence for studies concerned with the benefits of Tai Chi for rheumatoid arthritis (very low) (Mudano, Tugwell, Wells, \& Singh, 2019), chronic obstructive pulmonary disease (very low to moderate) (Ngai, Jones, \& Tam, 2016), and considerable heterogeneity between studies concerned with the prevention of cardiovascular disease (Hartley, Flowers, Lee, Ernst, \& Rees, 2014). Review authors have thus not been able to draw firm conclusions as to the health benefits of Tai Chi. However, not every review has reported such concerns, with high-quality evidence reported for trials testing the benefits of Tai Chi on pain, stiffness, and function in patients with osteoarthritis (Yan et al., 2013).

A more recent scoping review of the benefits of Tai Chi for people with long-term health conditions separated the trials in favor and not in favor of Tai Chi (Easwaran et al., 2020). They found a trend for high-quality trials to have favorable results for Tai Chi. Of the 117 trials with favorable results, $84 \%$ were scored as being of moderate to high quality, whereas only $68 \%$ received such scores among the 101 trials with unfavorable results. The review authors reported highquality evidence to support the use of Tai Chi for patients on a range of outcomes with cancer, chronic obstructive pulmonary disease, heart failure, lower back pain, osteoarthritis, osteoporosis, Parkinson's disease, and stroke (Easwaran et al., 2020).

Therefore, overall, the evidence base for Tai Chi to improve health among various patient groups is limited by poor-quality evidence. Moving forward, one solution is more explicit reporting of the interventions used. One recommendation is the use of a tool that requires authors to document several aspects of interventions in order to enable greater transparency (Wu, MacDonald, \& Pescatello, 2016).

\section{Expected Adherence to Tai Chi Exercise Interventions}

Previous reviews have synthesized the adherence data for exercise interventions for the prevention of falls for both class-based and home-based programs (McPhate, Simek, \& Haines, 2013; Simek, McPhate, \& Haines, 2012). For class-based interventions, four were Tai Chi trials, with a median number of sessions attended including dropouts of $74 \%$ (range 70-86\%; Greenspan, Wolf, Kelley, \& O'Grady, 2007; Voukelatos, Cumming, Lord, \& Rissel, 2007; Wolf et al., 2003; Wu, Keyes, Callas, Ren, \& Bookchin, 2010). This was no different from the overall pooled estimate of adherence for all 18 trials included in the review $(74 \%, 95 \%$ CI $[67,80]$; McPhate et al., 2013).

Below, I synthesize for the first time the adherence data from the Tai Chi trials included in the current Cochrane review of exercise interventions for preventing falls among community-dwelling older adults (Sherrington et al., 2019) (see Table 2). Adherence data from six Tai Chi trials included in the review were available, which indicated a median Tai Chi class attendance of three quarters from older adults (Li, Harmer, Fisher, \& McAuley, 2004; Taylor et al., 2012; Voukelatos et al., 2007; Wolf et al., 2003; Woo et al., 2007): $76 \%$ (range: $63-81 \%$ ) if excluded or $74 \%$ (range: $35-81 \%$ ) if included a study that had adherence rates that were much lower compared with all the other studies with a median of $63 \%$ attendance in the first 24 weeks and only $35 \%$ over the full 48 weeks (Day et al., 2015). Other data suggest that $53 \%$ (range: $47.0-58.6 \%$ ) of participants will attend at least $80 \%$ of the sessions (Logghe et al., 2009; Voukelatos et al., 2007) and that 79\% (range: $78.8-80.0 \%$ ) will attend at least $50 \%$ of the sessions (Li et al., 2004; Voukelatos et al., 2007). Although the Tai Chi exercise interventions were provided for a range of 16 weeks to 12 months, the adherence rates appear to be stable over time. Data suggest a median adherence rate of $71-81 \%$ from 16 weeks to 52 weeks and similar proportions (range: 78.8-80.0\%) that attend at least $50 \%$ of sessions from 16 to 26 weeks. Of those that attend at least $80 \%$ of sessions, data were only available from 13 to 16 weeks. These adherence rates appear broadly similar to those previously reported for exercise interventions, from $83 \%$ at 2 months and decreasing over time to $73 \%$ at 12 months (Nyman \& Victor, 2012).

For adherence to home-based exercise, a previous review suggested that the overall pooled estimate of adherence from all 23 trials was $21 \%(95 \%$ CI [15\%, 29\%], range: 0-68\%), although a high degree of between-study heterogeneity was reported (Simek et al., 2012). For programs that included a Tai Chi exercise component, there was no significant difference in the adherence levels (whether in the primary or sensitivity analysis for both full [proportion at $100 \%$ ] or partial adherence [proportion at 50\%]). When a Tai Chi exercise component was combined with a group exercise component, falls prevention education, home hazard assessment, or other multifactorial intervention, then there was only one significant difference: a Tai Chi exercise component plus a group exercise component resulted in a significantly lower adherence level in the partial adherence primary analysis $(\mathrm{OR}=0.49,95 \% \mathrm{CI}[0.25,0.96])$, which was no longer significant in the sensitivity analysis that excluded dropouts (Simek et al., 2012). However, these analyses were limited, because in two instances the home-based intervention was ancillary to the classbased component (Barnett, Smith, Lord, Williams, \& Baumand, 2003) _ for example, only requiring $30 \mathrm{~min}$ of home practice per week (Logghe et al., 2009). Indeed, the reviewers found that overall, partial adherence to home-based exercise was higher in programs without a class-based component (Simek et al., 2012). A 
Table 2 Adherence Data Reported by the Tai Chi Trials Included in the Current Cochrane Review of Exercise Interventions for Preventing Falls Among Community-Dwelling Older Adults (Sherrington et al., 2019)

\begin{tabular}{llll}
\hline Author (date) & Length of intervention & Average class attendance & Notes \\
\hline Voukelatos et al. (2007) & 16 weeks & $71 \%$ & 60 min once a week \\
Taylor et al. (2012) & 20 weeks & $79 \%(\mathrm{IQR}=49-90 \%)$ & Weekly sessions \\
& & $72 \%(\mathrm{IQR}=44-88 \%)$ & Twice-weekly sessions \\
Li et al. (2004) & 6 months & $78.21 \%$ & 61 out of 78 sessions, range $30-77$ \\
Day et al. (2015) & 48 weeks & $63 \%$ (median) & $\begin{array}{l}\text { Mean }(S D)=25.8(15.9) \text { out of } 48 \\
\text { in first } 24 \text { weeks } \\
\end{array}$ \\
& & $35 \%$ (median) & Mean $(S D)=34.4(26.9)$ out of 96 for the full 48 weeks \\
Wolf et al. (2003) & 48 weeks & $76 \%(\mathrm{SD}=19 \%)$ & 60 min twice a week building up to \\
& & 90 min twice a week for 48 weeks \\
Woo et al. (2007) & 12 months & Classes three times per week
\end{tabular}

Note. $\mathrm{IQR}=$ interquartile range.

further particular limitation of home-based exercise programs, is that frequency and type of exercise are only part of the equation when considering how much exercise one must do to gain the benefits; therefore, the above estimates are missing estimations of intensity and duration (Farlie, Ganz, \& Haines, 2018).

In relation to adherence, it is notable that different types of programs have been tested. One trial reported similar median adherence rates to Tai Chi classes provided once (79\%) or twice (72\%) a week, and so was able to provide a higher dose of Tai Chi with two classes per week (Taylor et al., 2012). Another study found higher adherence levels to class and video conference-based Tai Chi relative to simply providing a DVD for home practice $(\mathrm{Wu}$ et al., 2010). Practitioners can also draw on research into behavior change techniques in order to promote adherence to exercise (Zubala et al., 2017) including people with dementia (Nyman, Adamczewska, \& Howlett, 2018).

\section{Practical Implications}

Tai Chi can be highly effective for preventing falls. Tai Chi is a safe and accessible form of exercise. Indeed, over 500 trials and 120 systematic reviews have been conducted on the health benefits of Tai Chi and no studies have found that Tai Chi worsens a condition (Huston \& McFarlane, 2016). In addition, a systematic review of adverse event reporting from Tai Chi trials found Tai Chi is unlikely to result in serious adverse events but may result in minor musculoskeletal aches and pains (Wayne, Berkowitz, Litrownik, Buring, \& Yeh, 2014). For older adults with osteoporosis with vertebral fractures, it is recommended that they first receive advice on spine sparing techniques and that they avoid repetitive, weighted, or rapid end range flexion or extension of the spine (Skelton \& Mavroeidi, 2018). Frailer older adults, those with a high falls risk or multiple comorbid conditions affecting balance or strength, are recommended to only practice Tai Chi under the supervision of an instructor trained in adapting and tailoring the exercise prescription (Skelton \& Mavroeidi, 2018).

There is, however, a complication in the promotion of Tai Chi, which is the heterogeneity of Tai Chi practice. This concerns both styles of Tai Chi and styles of instructors. Styles of Tai Chi include Chen, Yang, Wu, Sun, and Hao, all of which have their own unique features and characteristics but share a common core set of principles. This means that it is possible that some styles of Tai Chi may be more effective for preventing falls than others. Indeed, the authors of one systematic review concluded that Yang style is likely to be more effective than Sun style Tai Chi (Huang et al., 2017). However, this was based on the evidence from three trials using Sun style. One trial found an effect at 24 weeks but not at 16 weeks, which was different from the number reported by the review authors (Voukelatos et al., 2007). Another trial that had a null effect that may be explained, as acknowledged by the trial authors, by insufficient intervention intensity, low class attendance, and attrition bias in the intervention group (Day et al., 2015). The final trial, as noted above, had a long-term follow-up, and because $60 \%$ of the sample continued with exercise after the interventions ceased, the similar reductions in fall rates across trial arms may have been the result of exercise conducted outside the interventions (Taylor et al., 2012). Further research could investigate whether certain features of particular styles of Tai Chi lend themselves to more effective prevention of falls. Though, the high level of overlap between styles, due to their common core, may give cause for a very small effect size between Tai Chi styles.

Of more importance than the style of Tai Chi is heterogeneity in styles of the instructors. Because Tai Chi originates in part from the martial arts, it does not have nationally recognized formal accreditation systems like other forms of professional training, such as for physiotherapy. Instructors have different levels of qualification and experience, different interpretations and emphases within styles, different teaching styles, and some may mix Tai Chi with other exercise, such as strength and balance training. While heterogeneity in the styles of the instructors is not unique to Tai Chi, it is a greater issue with Tai Chi interventions relative to exercise interventions delivered by physiotherapists and exercise instructors that are governed by national accreditation systems. Therefore, as with other interventions, Tai Chi exercise interventions used in future research will need to be manualized and standardized. Such standardization would need to be made widely available to the public to fully implement an evidence-based Tai Chi exercise intervention.

\section{Conclusion}

Tai Chi, the ancient Chinese traditional mind-body exercise, has attracted much research into its health benefits. Based on the foregoing evidence, one can conclude that Tai Chi is effective in preventing falls among older adults in the community and among various populations, including those at risk of falls, that have 
Parkinson's disease, or that have experienced a stroke. However, as with exercise interventions in general, there is no clear evidence of benefit for preventing falls in care homes and an absence of evidence with regard to rehabilitation on hospital wards. The few studies that have compared Tai Chi with other forms of exercise suggest that Tai Chi may be more effective in providing cognitive benefits for older adults and therefore may be more effective in preventing falls through this mechanism. Unfortunately, much of the related research with other patient groups and other health outcomes has been limited by the low quality of trials. Tai Chi trials have attracted similar adherence rates of 71$81 \%$ compared to other exercise-based interventions for preventing falls. It is an inherently safe form of exercise and, therefore, can be promoted among older adults. However, heterogeneity in how Tai Chi is taught, with no formal standardization, means that until evidence-based interventions become widely available, the implementation of such evidence among populations will be variable.

\section{References}

Acton, M. (2009). Eternal spring: Taijiquan, qi gong, and the cultivation of health, happiness and longevity. London: Singing Dragon.

Adamczewska, N., Barrado-Martín, Y., Thomas, S., Thomas, P., Vassallo, M., \& Nyman, S.R. (2019). Feasibility and accuracy of different methods for collecting data on falls among people with dementia. Alzheimer Disease \& Associated Disorders. Advance online publication. PubMed ID: 31789633 doi:10.1097/WAD.0000000000000364

Barnett, A., Smith, B., Lord, S.R., Williams, M., \& Baumand, A. (2003). Community-based group exercise improves balance and reduces falls in at-risk older people: A randomised controlled trial. Age and Ageing, 32(4), 407-414. PubMed ID: 12851185 doi:10.1093/ ageing/32.4.407

Burns, E.R., Stevens, J.A., \& Lee, R. (2016). The direct costs of fatal and non-fatal falls among older adults-United States. Journal of Safety Research, 58, 99-103. PubMed ID: 27620939 doi:10.1016/j.jsr. 2016.05.001

Cameron, I., Dyer, S., Panagoda, C., Murray, G., Hill, K., Cumming, R., \& Kerse, N. (2018). Interventions for preventing falls in older people in care facilities and hospitals (Review). Cochrane Database of Systematic Reviews, (9), CD005465. PubMed ID: 23235623 doi:10. 1002/14651858.cd005465.pub3

Campbell, A.J., \& Robertson, M.C. (2007). Rethinking individual and community fall prevention strategies: A meta-regression comparing single and multifactorial interventions. Age and Ageing, 36(6), 656662. PubMed ID: 18056731 doi:10.1093/ageing/afm122

Chang, Y.K., Nien, Y.H., Tsai, C.L., \& Etnier, J.L. (2010). Physical activity and cognition in older adults: The potential of Tai Chi chuan. Journal of Aging and Physical Activity, 18(4), 451-472. doi:10.1123/ japa.18.4.451

Choi, J., Moon, J., \& Song, R. (2005). Effects of Sun-style Tai Chi exercise on physical fitness and fall prevention in fall-prone older adults. Journal of Advanced Nursing, 51(2), 150-157. doi:10.1111/j.13652648.2005.03480.x

Day, L., Hill, K., Stathakis, V., Flicker, L., Segal, L., Cicuttini, F., \& Jolley, D. (2015). Impact of Tai-Chi on falls among preclinically disabled older people. A randomized controlled trial. Journal of the American Medical Directors Association, 16(5), 420-426. doi:10. 1016/j.jamda.2015.01.089

Del-Pino-Casado, R., Obrero-Gaitán, E., \& Lomas-Vega, R. (2016). The effect of Tai Chi on reducing the risk of falling: A systematic review and meta-analysis. American Journal of Chinese Medicine, 44(5), 895-906. doi:10.1142/S0192415X1650049X

Department of Health and Social Care. (2019). UK Chief Medical Officers' physical activity guidelines. London, UK: Author.

Easwaran, K., Gopalasingam, Y., Green, D.D., Lach, V., Melnyk, J.A., Wan, C., \& Bartlett, D.J. (2020). Effectiveness of Tai Chi for health promotion for adults with health conditions: A scoping review of meta-analyses. Disability and Rehabilitation. Advance online publication. doi:10.1080/09638288.2020.1725916

Faber, M.J., Bosscher, R.J., Chin, A.P.M.J., \& van Wieringen, P.C. (2006). Effects of exercise programs on falls and mobility in frail and pre-frail older adults: A multicenter randomized controlled trial. Archives of Physical Medicine and Rehabilitation, 87(7), 885-896. PubMed ID: 16813773 doi:10.1016/j.apmr.2006.04.005

Farlie, M., Ganz, D., \& Haines, T. (2018). Principles of physical activity promotion among older people. In S. R. Nyman, A. Barker, T. Haines, K. Horton, C. Musselwhite, G. Peeters, C. Victor, \& J. Wolff (Eds.), The Palgrave handbook of ageing and physical activity promotion (pp. 83-101). London: Palgrave MacMillan.

Foster, C., Armstrong, M., Hillsdon, M., Skelton, D., Mavroeidi, A., Cavill, N., \& Milton, K. (2018). Muscle and bone strengthening and balance activities for general health benefits in adults and older adults: Summary of a rapid evidence review for the UK Chief Medical Officers' update of the physical activity guidelines. London: Public Health England.

Gillespie, L.D., Robertson, M.C., Gillespie, W.J., Sherrington, C., Gates, S., Clemson, L.M., \& Lamb, S.E. (2012). Interventions for preventing falls in older people living in the community. Cochrane Database of Systematic Reviews, (9), CD007146. PubMed ID: 22972103 doi:10. 1002/14651858.cd007146.pubc3

Greenspan, A. I., Wolf, S.L., Kelley, M.E., \& O’Grady, M. (2007). Tai Chi and perceived health status in older adults who are transitionally frail: A randomized controlled trial. Physical Therapy, 87(5), 525-535. PubMed ID: 17405808 doi:10.2522/ptj.20050378

Hackney, M.E., \& Wolf, S.L. (2014). Impact of Tai Chi Chu' an practice on balance and mobility in older adults: An integrative review of 20 years of research. Journal of Geriatric Physical Therapy, 37(3), 127 135. doi:10.1519/JPT.0b013e3182abe784

Hartley, L., Flowers, N., Lee, M.S., Ernst, E., \& Rees, K. (2014). Tai Chi for primary prevention of cardiovascular disease. Cochrane Database of Systematic Reviews, (4), Cd010366. PubMed ID: 24715694 doi:10. 1002/14651858.cd010366.pub2

Huang, Z., Feng, Y., Li, Y., \& Lv, C. (2017). Systematic review and metaanalysis: Tai Chi for preventing falls in older adults. BMJ Open, 7(2), e013661. doi:10.1136/bmjopen-2016-013661

Huston, P., \& McFarlane, B. (2016). Health benefits of Tai Chi: What is the evidence? Canadian Family Physician, 62(11), 881-890. PubMed ID: 28661865

Hwang, H., Cheng, S., Lee-Hsieh, J., Chien, D., Chen, C., \& Lin, M. (2016). Effects of home-based Tai Chi and lower extremity training and selfpractice on falls and functional outcomes in older fallers from the emergency department-A randomized controlled trial. Journal of the American Geriatrics Society, 64(3), 518-525. doi:10.1111/jgs.13952

Lee, L.Y., Lee, D.T., \& Woo, J. (2010). The psychosocial effect of Tai Chi on nursing home residents. Journal of Clinical Nursing, 19(7-8), 927-938. doi:10.1111/j.1365-2702.2009.02793.x

Li, F., Harmer, P., \& Chou, L.S. (2019). Dual-task walking capacity mediates Tai Ji Quan impact on physical and cognitive function. Medicine \& Science in Sports \& Exercise, 51(11), 2318-2324. PubMed ID: 31169795 doi:10.1249/MSS.0000000000002051

Li, F., Harmer, P., Eckstrom, E., Fitzgerald, K., Chou, L.S., \& Liu, Y. (2019). Effectiveness of Tai Ji Quan vs multimodal and stretching 
exercise interventions for reducing injurious falls in older adults at high risk of falling: Follow-up analysis of a randomized clinical trial. JAMA Network Open, 2(2), e188280. PubMed ID: 30768195 doi:10. 1001/jamanetworkopen.2018.8280

Li, F., Harmer, P., Fisher, K.J., \& McAuley, E. (2004). Tai Chi: Improving functional balance and predicting subsequent falls in older persons. Medicine \& Science in Sports \& Exercise, 36(12), 2046-2052. doi:10.1249/01.MSS.0000147590.54632.E7

Li, F., Harmer, P., Fitzgerald, K., Eckstrom, E., Akers, L., Chou, L.-S., . . . Winters-Stone, K. (2018). Effectiveness of a therapeutic Tai Ji Quan intervention vs a multimodal exercise intervention to prevent falls among older adults at high risk of falling: A randomized clinical trial. JAMA Internal Medicine, 178(10):1301-1310. PubMed ID: 30208396 doi:10.1001/jamainternmed.2018.3915

Li, J., Hong, Y., \& Chan, K. (2001). Tai Chi: Physiological characteristics and beneficial effects on health. British Journal of Sports Medicine, 35(3), 148-156. doi:10.1136/bjsm.35.3.148

Li, Q., Liu, J., Dai, F., \& Dai, F. (2020). Tai Chi versus routine exercise in patients with early- or mild-stage Parkinson's disease: A retrospective cohort analysis. Brazilian Journal of Medical and Biological Research, 53(2), e9171. PubMed ID: 32049101 doi:10.1590/1414$431 \times 20199171$

Liu, H., \& Frank, A. (2010). Tai Chi as a balance improvement exercise for older adults: A systematic review. Journal of Geriatric Physical Therapy, 33(3), 103-109. PubMed ID: 21155504

Logghe, I.H., Verhagen, A.P., Rademaker, A.C., Bierma-Zeinstra, S.M., van Rossum, E., Faber, M.J., \& Koes, B.W. (2010). The effects of Tai Chi on fall prevention, fear of falling and balance in older people: A meta-analysis. Preventive Medicine, 51(3-4), 222-227. PubMed ID: 20558197 doi:10.1016/j.ypmed.2010.06.003

Logghe, I.H., Zeeuwe, P.E., Verhagen, A.P., Wijnen-Sponselee, R.M., Willemsen, S.P., Bierma-Zeinstra, S.M., ... Koes, B.W. (2009). Lack of effect of Tai Chi chuan in preventing falls in elderly people living at home: A randomized clinical trial. Journal of the American Geriatrics Society, 57(1), 70-75. PubMed ID: 19054193 doi:10. 1111/j.1532-5415.2008.02064.x

Lomas-Vega, R., Obrero-Gaitán, E., Molina-Ortega, F., \& Del-PinoCasado, R. (2017). Tai Chi for risk of falls. A meta-analysis. Journal of the American Geriatrics Society, 65(9), 2037-2043. doi:10.1111/ jgs. 15008

Martin, F.C. (2011). Falls risk factors: Assessment and management to prevent falls and fractures. Canadian Journal on Aging, 30(1), 33-44. doi:10.1017/S0714980810000747

McPhate, L., Simek, E.M., \& Haines, T.P. (2013). Program-related factors are associated with adherence to group exercise interventions for the prevention of falls: A systematic review. Journal of Physiotherapy, 59(2), 81-92. doi:10.1016/S1836-9553(13)70160-7

Mudano, A.S., Tugwell, P., Wells, G.A., \& Singh, J.A. (2019). Tai Chi for rheumatoid arthritis. Cochrane Database of Systematic Reviews, (9), Cd004849. PubMed ID: 31553478 doi:10.1002/14651858. cd004849.pub2

Ng, C., Fairhall, N., Wallbank, G., Tiedemann, A., Michaleff, Z.A., \& Sherrington, C. (2019). Exercise for falls prevention in communitydwelling older adults: Trial and participant characteristics, interventions and bias in clinical trials from a systematic review. BMJ Open Sport \& Exercise Medicine, 5(1), e000663. PubMed ID: 31908838 doi:10.1136/bmjsem-2019-000663

Ngai, S.P., Jones, A.Y., \& Tam, W.W. (2016). Tai Chi for chronic obstructive pulmonary disease (COPD). Cochrane Database of Systematic Reviews, (6), Cd009953. PubMed ID: 27272131 doi:10.1002/14651858.cd009953.pub2
NHS Choices. (2018). A guide to Tai Chi. Retrieved from http://www.nhs. uk/Livewell/fitness/Pages/taichi.aspx

Ni, M., Mooney, K., Richards, L., Balachandran, A., Sun, M., Harriell, K., . . Signorile, J.F. (2014). Comparative impacts of Tai Chi, balance training, and a specially-designed yoga program on balance in older fallers. Archives of Physical Medicine and Rehabilitation, 95(9), 16201628.e30. PubMed ID: 24835753 doi:10.1016/j.apmr.2014.04.022

Nowalk, M., Prendergast, J., Bayles, C., D’Amico, F., \& Colvin, G. (2001). A randomized trial of exercise programs among older individuals living in two long-term care facilities: The FallsFREE program. Journal of the American Geriatrics Society, 49(7), 859865. doi:10.1046/j.1532-5415.2001.49174.x

Nyman, S.R., Adamczewska, N., \& Howlett, N. (2018). Systematic review of behaviour change techniques to promote participation in physical activity among people with dementia. British Journal of Health Psychology, 23(1), 148-170. doi:10.1111/bjhp.12279

Nyman, S.R., Ingram, W., Sanders, J., Thomas, P., Thomas, S., Vassallo, M., .. Barrado-Martín, Y. (2019). Randomised controlled trial of the effect of Tai Chi on postural balance of people with dementia. Clinical Interventions in Aging, 14, 2017-2029. PubMed ID: 31819385 doi:10.2147/CIA.S228931

Nyman, S.R., \& Skelton, D. (2017). The case for Tai Chi in the repertoire of strategies to prevent falls among older people. Perspect Public Health, 137(2), 85-86. PubMed ID: 28244837 doi:10.1177/ 1757913916685642

Nyman, S.R., \& Victor, C. (2012). Older people's participation in and engagement with falls prevention interventions in community settings: An augment to the Cochrane systematic review. Age and Ageing, 41(1), 16-23. doi:10.1093/ageing/afr103

Peel, N.M. (2011). Epidemiology of falls in older age. Canadian Journal on Aging, 30(1), 7-19. doi:10.1017/S071498081000070X

Rubenstein, L.Z. (2006). Falls in older people: Epidemiology, risk factors, and strategies for prevention. Age and Ageing, 35(Suppl. 2), ii37ii41. doi:10.1093/ageing/afl084

Samaras, N., Chevalley, T., Samaras, D., \& Gold, G. (2010). Older patients in the emergency department: A review. Annals of Emergency Medicine, 56(3), 261-269. doi:10.1016/j.annemergmed.2010.04.015

Saravanakumar, P., Higgins, I., van der Riet, P., Marquez, J., \& Sibbritt, D. (2014). The influence of Tai Chi and yoga on balance and falls in a residential care setting: A randomised controlled trial. Contemporary Nurse, 48(1), 76-87. doi:10.1080/10376178.2014.11081929

Sherrington, C., Fairhall, N., Wallbank, G., Tiedemann, A., Michaleff, Z., Howard, K., ... Lamb, S. (2019). Exercise for preventing falls in older people living in the community (Review). Cochrane Database of Systematic Reviews, 1(1), CD012424. PubMed ID: 30703272 doi:10.1002/14651858.CD012424.pub2

Sherrington, C., Michaleff, Z.A., Fairhall, N., Paul, S.S., Tiedemann, A., Whitney, J., . . Lord, S.R. (2017). Exercise to prevent falls in older adults: An updated systematic review and meta-analysis. British Journal of Sports Medicine, 51(24), 1750-1758. PubMed ID: 27707740 doi:10.1136/bjsports-2016-096547

Simek, E.M., McPhate, L., \& Haines, T.P. (2012). Adherence to and efficacy of home exercise programs to prevent falls: A systematic review and meta-analysis of the impact of exercise program characteristics. Preventive Medicine, 55(4), 262-275. doi:10.1016/j. ypmed.2012.07.007

Skelton, D., \& Mavroeidi, A. (2018). Which strength and balance activities are safe and efficacious for individuals with specific challenges (osteoporosis, vertebral fractures, frailty, dementia)?: A Narrative review. Journal of Frailty, Sarcopenia and Falls, 3(2), 85-104. doi:10.22540/JFSF-03-085 
Solloway, M., Taylor, S., Shekelle, P., Miake-Lye, I., Beroes, J., Shanman, R., \& Hempel, S. (2016). An evidence map of the effect of Tai Chi on health outcomes. Systematic Reviews, 5(1), e126. doi:10.1186/ s13643-016-0300-y

Son, N.-K., Ryu, Y.U., Jeong, H.-W., Jang, Y.-H., \& Kim, H.-D. (2016). Comparison of 2 different exercise approaches: Tai Chi versus otago, in community-dwelling older women. Journal of Geriatric Physical Therapy, 39(2), 51-57. doi:10.1519/JPT.0000000000000042

Speechley, M. (2011). Unintentional falls in older adults: A methodological historical review. Canadian Journal on Aging, 30(1), 21-32. doi:10.1017/S0714980810000735

Taylor, D., Hale, L., Schluter, P., Waters, D., Binns, E., McCracken, H., ... Wolf, S.L. (2012). Effectiveness of Tai Chi as a communitybased falls prevention intervention: A randomized controlled trial. Journal of the American Geriatrics Society, 60(5), 841-848. doi:10. 1111/j.1532-5415.2012.03928.x

Tousignant, M., Corriveau, H., Roy, P.M., Desrosiers, J., Dubuc, N., \& Hébert, R. (2013). Efficacy of supervised Tai Chi exercises versus conventional physical therapy exercises in fall prevention for frail older adults: A randomized controlled trial. Disability and Rehabilitation, 35(17), 1429-1435. doi:10.3109/09638288.2012. 737084

Tousignant, M., Corriveau, H., Roy, P.M., Desrosiers, J., Dubuc, N., Hébert, R., ... Beaudoin, A.J. (2012). The effect of supervised Tai Chi intervention compared to a physiotherapy program on fall-related clinical outcomes: A randomized clinical trial. Disability and Rehabilitation, 34(3), 196-201. doi:10.3109/09638288.2011. 591891

Voukelatos, A., Cumming, R.G., Lord, S.R., \& Rissel, C. (2007). A randomized, controlled trial of Tai Chi for the prevention of falls: The Central Sydney Tai Chi trial. Journal of the American Geriatrics Society, 55(8), 1185-1191. PubMed ID: 17661956 doi:10.1111/j. 1532-5415.2007.01244.x

Wayne, P., Berkowitz, D., Litrownik, D., Buring, J., \& Yeh, G. (2014). What do we really know about the safety of Tai Chi?: A systematic review of adverse event reports in randomized trials. Archives of Physical Medicine and Rehabilitation, 95(12), 2470-2483. doi:10. 1016/j.apmr.2014.05.005

Wayne, P., \& Fuerst, M. (2013). The Harvard medical school guide to Tai Chi: 12 weeks to a healthy body, strong heart \& sharp mind. Colorado: Shambhala.

Winser, S., Tsang, W., Krishnamurthy, K., \& Kannan, P. (2018). Does Tai Chi improve balance and reduce falls incidence in neurological disorders? A systematic review and meta-analysis.
Clinical Rehabilitation, 32(9), 1157-1168. doi:10.1177/ 0269215518773442

Wolf, S., Barnhart, H., Kutner, N., McNeely, E., Coogler, C., Xu, T., \& Group, A.F. (1996). Reducing frailty and falls in older persons: An investigation of Tai Chi and computerised balance training. Journal of the American Geriatrics Society, 44(5), 489-497. doi:10.1111/j. 1532-5415.1996.tb01432.x

Wolf, S., Sattin, R., Kutner, M., O’Grady, M., Greenspan, A., \& Gregor, R. (2003). Intense Tai Chi exercise training and fall occurrences in older, transitionally frail adults: A randomized, controlled trial. Journal of the American Geriatrics Society, 51(12), 1693-1701. doi:10.1046/j. 1532-5415.2003.51552.x

Woo, J., Hong, A., Lau, E., \& Lynn, H. (2007). A randomised controlled trial of Tai Chi and resistance exercise on bone health, muscle strength and balance in community-living elderly people. Age and Ageing, 36(3), 262-268. doi:10.1093/ageing/afm005

World Health Organization. (2007). WHO global report on falls prevention in older age. Geneva: Author.

Wu, G., Keyes, L., Callas, P., Ren, X., \& Bookchin, B. (2010). Comparison of telecommunication, community, and home-based Tai Chi exercise programs on compliance and effectiveness in elders at risk for falls. Archives of Physical Medicine and Rehabilitation, 91(6), 849-856. PubMed ID: 20510973 doi:10.1016/j.apmr.2010.01.024

Wu, Y., MacDonald, H.V., \& Pescatello, L.S. (2016). Evaluating exercise prescription and instructional methods used in Tai Chi studies aimed at improving balance in older adults: A systematic review. Journal of the American Geriatrics Society, 64(10), 2074-2080. PubMed ID: 2755009 doi:10.1111/jgs. 14242

Yan, J.H., Gu, W.J., Sun, J., Zhang, W.X., Li, B.W., \& Pan, L. (2013). Efficacy of Tai Chi on pain, stiffness and function in patients with osteoarthritis: A meta-analysis. PLoS One, 8(4), e61672. PubMed ID: 23620778 doi:10.1371/journal.pone.0061672

Zheng, P., Zhang, J., \& Tong, L. (2013). Meta analysis of the effect of Tai Chi on reducing falls among elders living at home. Chinese Journal of Modern Nursing, 19, 1123-1127.

Zhong, D., Xiao, Q., Xiao, X., Li, Y., Ye, J., Xia, L., . . J Jin, R. (2020). Tai Chi for improving balance and reducing falls: An overview of 14 systematic reviews. Annals of Physical and Rehabilitation Medicine. Advance online publication. doi:10.1016/j.rehab.2019.12.008

Zubala, A., MacGillivray, S., Frost, H., Kroll, T., Skelton, D.A., Gavine, A., ... Morris, J. (2017). Promotion of physical activity interventions for community dwelling older adults: A systematic review of reviews. PLoS One, 12(7), e0180902. PubMed ID: 28700754 doi:10.1371/ journal.pone.0180902 